\title{
Least-Restrictive Robust MPC of Periodic Affine Systems with Application to Building Climate Control
}

\author{
Ravi Gondhalekar, Frauke Oldewurtel, Colin N. Jones
}

\begin{abstract}
Robust state-feedback model predictive control (MPC) of discrete-time periodic affine systems is considered. States and inputs are subject to periodically time-dependent, hard, convex, polyhedral constraints. Disturbances are additive, bounded and subject to periodically time-dependent bounds. The control objective is given in terms of periodically timedependent costs. First, maximum robust periodic controlled invariant sets are formally characterized and subsequently employed in the design of least-restrictive robustly strongly feasible periodic MPC problems. Finally, the proposed methods are applied to controlling room temperatures in buildings.
\end{abstract}

Index Terms-Model predictive control; Constrained control; Set invariance; Periodic systems; Building climate control

\section{INTRODUCTION}

MPC of discrete-time periodic affine systems is considered in this paper. Periodic systems are useful extensions of time-invariant systems, and provide a natural framework for modeling various real-world phenomena and control problems $[3,25,26,29]$. The presented methods are an extension of theory developed for periodic linear systems in [14,15]. Periodic affine systems are an important generalization of periodic linear systems, but many tools for rigorously dealing with periodic affine systems can be derived from the tools for periodic linear systems. Thus in this paper the conservativeness and complication of periodic MPC methods designed for general nonlinear periodic systems is avoided [7,28].

A key point of this paper is the characterization of maximum robust periodic controlled invariant sets (Def. 10) and their use in robust periodic MPC problem formulations. Maximum robust periodic controlled invariant sets provide a necessary and sufficient condition for the existence of an infinite admissible control input trajectory [5], and are employed in this paper to least-restrictively enforce robust strong feasibility (Def. 7) of periodic MPC problems. Thus constraints can be rigorously enforced, indefinitely, for any

Ravi Gondhalekar is with the Department of Mechanical Engineering and Frontier Research Base for Global Young Researchers, Graduate School of Engineering, Osaka University, Japan. Ravi gratefully acknowledges funding received through the Program of Promotion of Environmental Improvement to Enhance Young Researchers' Independence under the Special Coordination Funds for Promoting Science and Technology, Japan Ministry of Education, Culture, Sports, Science and Technology. ravi.gondhalekar@wakate.frc.eng.osaka-u.ac.jp

Frauke Oldewurtel and Colin N. Jones are with the Automatic Control Laboratory, Department of Electrical Engineering, Swiss Federal Institute of Technology in Zurich (ETHZ), Switzerland. Frauke and Colin gratefully acknowledge funding received through the Automatic Control Laboratory. Swisselectric Research, CCEM-CH and Siemens Building Technologies are gratefully acknowledged for their financial support of the OptiControl project about energy efficient building climate control. \{oldewurtel, jones\}@control.ee.ethz.ch prediction horizon length, even one shorter than one period length. Closely related maximum periodic controlled invariant sets for periodic linear systems were considered in $[5,14,15]$. MPC was not considered in [5]. Unlike [14,15], in this paper robustness is considered. Robustness in MPC is usually enforced either by considering min-max openloop predictions [2] or by performing closed-loop predictions $[1,2,11,16,24]$. The former generally results in restrictive and conservative control laws. The latter is generally less restrictive and conservative than the former, but results in larger computational complexity. The approach proposed here performs the open-loop prediction of usual nominal MPC formulations (not following the min-max paradigm), but robust strong feasibility is least-restrictively enforced by a constraint on the first prediction step. Thus the computational complexity is lower than with closed-loop prediction MPC, but by exploiting the necessary and sufficient condition for recursive feasibility provided by the maximum robust periodic controlled invariant set, a periodic MPC problem with no conservativeness with respect to the controllable state-space is derived. Least-restrictively enforcing strong feasibility of MPC problems by invariant set constraints on states prior to the terminal prediction state was previously considered in $[12,13]$. Note that the proposed methods are directly applicable to time-invariant MPC formulations also.

Periodic MPC seems appropriate for many real-world control problems, yet it appears little has been done in this field. The reader is referred to $[6,7,20,21,23] /[7,28]$ on MPC of periodic linear/nonlinear systems. A main objective of this paper is to demonstrate the power and flexibility of periodic MPC problem formulations via the example of building climate control. Almost $40 \%$ of the electric energy generated globally is consumed in the residential and commercial sectors [19], thus building climate control is receiving much attention in the fight to reduce energy costs and consumption. The goal is to minimize the energy cost of heating and cooling a building's rooms, while satisfying temperature constraints, imposed for occupants' comfort. The use of MPC for building climate control $[17,18,27]$ is motivated by the fact that the plant is heavily influenced by external factors that can, to some extent, be forecast, e.g. the weather and occupants' behavior. The dynamics of the building itself are time-invariant, but the control problem is fundamentally periodic, with a short-term period length of one day, and a seasonal period length of one year (the latter is ignored in this paper). By employing a periodic MPC framework many details about the plant and control objective can be naturally encoded within the model. For example, the fluctuations 
of activities of office workers (assumed mostly active from $8 \mathrm{am}$ to $6 \mathrm{pm}$ ) and the outside air temperature (usually higher during the day than during the night) is periodically timedependent and closely predictable. However, predictions are not perfect, and the system is subject to stochastic disturbances, for example because clouds affect the intensity of incident solar radiation. However, these stochastic disturbances are bounded with periodically time-dependent bounds depending on the time of day. Also, the control objective is periodically time-dependent, for example because energy pricing policy prescribes different tariffs for day versus night. Finally, it is highly desirable to accommodate periodically time-dependent constraints, because for an office the temperature constraints need only be enforced during business hours, and relaxing the constraints outside business hours facilitates lower energy use. Some periodic details can straightforwardly be included within an LTI-MPC framework. However, periodically time-dependent hard constraints cannot be enforced rigorously and recursively in an LTI-MPC framework without introducing conservativeness, and are not least-restrictively enforceable without using the maximum robust periodic controlled invariant sets proposed here.

Notation: The set of reals is denoted by $\mathbb{R}$, the set of nonnegative integers by $\mathbb{N}\left(\mathbb{N}_{+}:=\mathbb{N} \backslash\{0\}\right)$, the set of consecutive non-negative integers $\{j, \ldots, k\}$ by $\mathbb{N}_{j}^{k}$. For sets $\mathbb{A}, \mathbb{B} \subseteq \mathbb{R}^{n}$ let $\mathbb{A} \ominus \mathbb{B}:=\left\{x \in \mathbb{R}^{n} \mid x+b \in \mathbb{A} \forall b \in \mathbb{B}\right\}$. Let $\bmod : \mathbb{N} \times$ $\mathbb{N}_{+} \rightarrow \mathbb{N}, \bmod (i, j):=\min _{k \in \mathbb{N}}\{i-k j \mid i-k j \geq 0\}$. Denote by $I_{n}$ the $n \times n$ identity matrix, by $0_{\{n, m\}}$ the $n \times m$ zero matrix, by 0 the zero matrix with appropriate dimension, and by $\operatorname{diag}\left(a_{1}, \ldots, a_{n}\right)$ the block-diagonal matrix of $n$ elements $a_{i}$. A sequence of elements $x_{i} \in \mathbb{X} \forall i \in \mathbb{N}_{j}^{k}$ is denoted by $\left\{x_{i} \in \mathbb{X}\right\}_{i=j}^{k}$. Let $\psi_{(i, k)}$ denote the future value of $\psi$ at step $i+k$, as predicted from step $i$. Let $\psi_{i}:=\psi_{(i, 0)}$. 'Polytope' refers to a closed, bounded and convex intersection of a finite number of half-spaces.

\section{Periodic MPC Control Law Design}

\section{A. Problem Setting}

We consider discrete-time periodic affine system

$$
x_{i+1}=A_{j} x_{i}+B_{j} u_{i}+C_{j} w_{i}+c_{j}
$$

with step $i \in \mathbb{N}$, period length $p \in \mathbb{N}_{+}$, inter-period stepindex $j:=\bmod (i, p) \in \mathbb{N}_{0}^{p-1}$, state $x \in \mathbb{R}^{n}$, control input $u \in \mathbb{R}^{m}$, disturbance $w \in \mathbb{R}^{v}$ and $n, m, v \in \mathbb{N}_{+}$. State and control input are required to satisfy

$$
E_{j} x_{i}+G_{j} u_{i} \leq W_{j} \quad \forall i \in \mathbb{N} .
$$

Entities $A_{j}, B_{j}, C_{j}, c_{j}, E_{j}, G_{j}$ and $W_{j}$ have compatible dimensions for all $j \in \mathbb{N}_{0}^{p-1}$.

Assumption 1: Disturbances satisfy $w_{i} \in \mathbb{W}_{j} \forall i \in \mathbb{N}$, where $\mathbb{W}_{j} \subset \mathbb{R}^{v} \forall j \in \mathbb{N}_{0}^{p-1}$ are polytopes with non-empty interiors and satisfy $0 \in \mathbb{W}_{j} \forall j \in \mathbb{N}_{0}^{p-1}$.

Let $r_{j} \in \mathbb{R}^{n} \forall j \in \mathbb{N}_{0}^{p-1}$ denote the state reference trajectory, over one period. For initial state $x_{k}$ the control objective is to minimize the quadratic cost function

$$
\begin{aligned}
V & :=\sum_{i=k}^{\infty}\left[\mathcal{L}_{\mathrm{x}}\left(x_{i}, i\right)+\mathcal{L}_{\mathrm{u}}\left(u_{i}, i\right)\right] \\
\mathcal{L}_{\mathrm{x}}(x, i) & :=\left(x-r_{\bmod (i, p)}\right)^{\top} Q_{\bmod (i, p)}\left(x-r_{\bmod (i, p)}\right) \\
\mathcal{L}_{\mathrm{u}}(u, i) & :=R_{\bmod (i, p)} u
\end{aligned}
$$

with $Q_{j} \in \mathbb{R}^{n \times n}, Q_{j} \succeq 0, R_{j} \in \mathbb{R}^{1 \times m} \forall j \in \mathbb{N}_{0}^{p-1}$.

\section{B. A Basic Periodic MPC Problem}

MPC achieves closed-loop control of system (1) subject to (2) by applying at each state $x_{i}=x_{(i, 0)}$ the first control input $u_{(i, 0)}$ of predicted control input trajectory $U_{i}^{*}\left(x_{i}\right):=$ $\left[u_{(i, 0)}^{\top}, \ldots, u_{(i, N-1)}^{\top}\right]^{\top} \in \mathbb{R}^{N m}$ given by Problem 3.

Remark 2: Prediction state constraint sets $\mathbb{X}_{j}, \mathbb{Y}_{j} \subseteq \mathbb{R}^{n}$ $\forall j \in \mathbb{N}_{0}^{p-1}$ of Eqs. (5) and (6) are defined in Section II-D to yield periodic MPC control laws with desirable properties.

Problem 3: Determine

$$
\begin{aligned}
U_{i}^{*}\left(x_{i}\right):= & \arg \min _{U_{i} \in \mathbb{U}\left(x_{i}\right)} J\left(x_{i}, U_{i}\right) \\
J\left(x_{i}, U_{i}\right):= & \sum_{k=0}^{N-1}\left[\mathcal{L}_{\mathrm{x}}\left(x_{(i, k)}, i+k\right)+\mathcal{L}_{\mathrm{u}}\left(u_{(i, k)}, i+k\right)\right] \\
& +\mathcal{L}_{\mathrm{x}}\left(x_{(i, N)}, i+N\right) \\
\mathbb{U}\left(x_{i}\right):= & \left\{U_{i} \in \mathbb{R}^{N m} \mid\right. \\
& E_{\bmod (i+k, p)} x_{(i, k)}+G_{\bmod (i+k, p)} u_{(i, k)} \\
\leq & W_{\bmod (i+k, p)} \quad \forall k \in \mathbb{N}_{0}^{N-1} \\
& x_{(i, 1)} \in \mathbb{X}_{\bmod (i+1, p)} \\
& \left.x_{(i, k)} \in \mathbb{Y}_{\bmod (i+k, p)} \quad \forall k \in \mathbb{N}_{2}^{N}\right\} \\
x_{(i, k+1)} & A_{\bmod (i+k, p)} x_{(i, k)}+B_{\bmod (i+k, p)} u_{(i, k)} \\
& +c_{\bmod (i+k, p)} \forall k \in \mathbb{N}_{0}^{N-1} .
\end{aligned}
$$

The periodic MPC control law resulting from MPC Problem 3 is given implicitly by $\kappa\left(x_{i}\right):=F U_{i}^{*}\left(x_{i}\right), F:=$ $\left[I_{m}, 0_{\{m,(N-1) m\}}\right]$. The closed-loop state trajectory evolves according to $x_{i+1}=A_{j} x_{i}+B_{j} \kappa\left(x_{i}\right)+C_{j} w_{i}+c_{j}$.

Remark 4: A more general terminal cost can be employed in Eq. (3). This detail is ignored here for simplicity.

Remark 5: Prediction dynamics (7) contain no term to take into account the additive disturbances. This is equivalent to open-loop MPC formulations with expectation $\mathrm{E}\left[w_{(i, k)}\right]=0$ $\forall(i, k) \in \mathbb{N} \times \mathbb{N}_{0}^{N-1}$. This circumnavigates the computational complexities of more advanced closed-loop prediction MPC strategies $[1,2,11,16,24]$. However, by suitable design of the prediction state constraint sets $\mathbb{X}_{j}$ and $\mathbb{Y}_{j}$ of Eqs. (5) and (6) (see Remark 2) in this paper we avoid the (often crippling) conservativeness induced by min-max open-loop prediction MPC [2], while rigorously enforcing robustness.

Definition 6: Sets $\mathbb{F}_{j}:=\left\{x_{j} \in \mathbb{R}^{n} \mid \mathbb{U}\left(x_{j}\right) \neq \emptyset\right\} \forall j \in \mathbb{N}_{0}^{p-1}$ are termed the sets of feasible states of MPC Problem 3, and at step $i$ any state $x_{i} \in \mathbb{F}_{j}$ is termed a feasible state.

Definition 7: MPC Problem 3 is robustly strongly feasible if and only if:

$$
\begin{aligned}
x_{i+1}= & A_{j} x_{i}+B_{j} F U_{i}+C_{j} w_{i}+c_{j} \in \mathbb{F}_{\bmod (i+1, p)} \\
& \forall w_{i} \in \mathbb{W}_{j} \quad \forall U_{i} \in \mathbb{U}\left(x_{i}\right) \quad \forall i \in \mathbb{N}_{k}^{\infty} \\
& \forall x_{k} \in \mathbb{F}_{k} \quad \forall k \in \mathbb{N}_{0}^{p-1} .
\end{aligned}
$$


The above expression states that the closed-loop state trajectory $\left\{x_{i}\right\}_{i=k}^{\infty}$ from any feasible initial state $x_{k} \in \mathbb{F}_{k}$, due to any sequence $\left\{U_{i} \in \mathbb{U}\left(x_{i}\right)\right\}_{i=k}^{\infty}$ of feasible solutions to MPC Problem 3, and due to any sequence $\left\{w_{i} \in \mathbb{W}_{j}\right\}_{i=k}^{\infty}$ of admissible disturbance realizations, remains within the sets $\mathbb{F}_{j}$ of feasible states indefinitely.

The design parameters of MPC Problem 3 are prediction horizon length $N$, stage cost matrices $Q_{j}, R_{j}$, and prediction state constraint sets $\mathbb{X}_{j}, \mathbb{Y}_{j}$ for all $j \in \mathbb{N}_{0}^{p-1}$. For compact notation denote by $\mathbb{D}$ a realization of all design parameters of MPC Problem 3.

Definition 8: Suppose a specific realization $\hat{\mathbb{D}}$ of design parameters of MPC Problem 3 results in sets $\hat{\mathbb{F}}_{j} \subseteq \mathbb{R}^{n}$ $\forall j \in \mathbb{N}_{0}^{p-1}$ of feasible states. Then MPC Problem 3 with the design parameters of $\hat{\mathbb{D}}$ is termed a least-restrictive robustly strongly feasible MPC problem if and only if it is robustly strongly feasible according to Definition 7, and in addition any other realization $\mathbb{D}$ of design parameters in MPC Problem 3 results in $\mathbb{F}_{j} \subseteq \hat{\mathbb{F}}_{j} \forall j \in \mathbb{N}_{0}^{p-1}$.

\section{Robust Periodic Controlled Set Invariance}

Robust periodic controlled invariance of discrete-time periodic affine system (1) is proposed and characterized in this section. The derived sets are employed in Section II-D to enforce desirable properties in MPC Problem 3.

Definition 9: A set $\left\{\mathbb{C}_{0}, \ldots, \mathbb{C}_{p-1}\right\}$ of sets $\mathbb{C}_{j} \subseteq \mathbb{R}^{n} \forall j \in$ $\mathbb{N}_{0}^{p-1}$ is a robust periodic controlled invariant set of system (1) if and only if:

$$
\begin{array}{r}
\exists \kappa_{j}: \mathbb{R}^{n} \rightarrow \mathbb{R}^{m} \text { s.t. } E_{j} x+G_{j} \kappa_{j}(x) \leq W_{j} \\
\wedge \quad A_{j} x+B_{j} \kappa_{j}(x)+C_{j} w+c_{j} \in \mathbb{C}_{\bmod (j+1, p)} \\
\forall w \in \mathbb{W}_{j} \quad \forall x \in \mathbb{C}_{j} \quad \forall j \in \mathbb{N}_{0}^{p-1} .
\end{array}
$$

Definition 10: The maximum robust periodic controlled invariant set is the set $\left\{\mathbb{C}_{0}^{*}, \ldots, \mathbb{C}_{p-1}^{*}\right\}$ of sets $\mathbb{C}_{j}^{*}$ as follows:

$$
\begin{aligned}
\mathbb{C}_{j}^{*}:= & \left\{x_{j} \in \mathbb{R}^{n} \mid \exists \kappa_{l}: \mathbb{R}^{n} \rightarrow \mathbb{R}^{m} \quad \forall l \in \mathbb{N}_{0}^{p-1}\right. \text { s.t. } \\
& x_{i+1}=A_{k} x_{i}+B_{k} \kappa_{k}\left(x_{i}\right)+C_{k} w_{i}+c_{k} \wedge \\
& E_{k} x_{i}+G_{k} \kappa_{k}\left(x_{i}\right) \leq W_{k} \wedge k=\bmod (i, p) \\
& \left.\forall w_{i} \in \mathbb{W}_{k} \forall i \in \mathbb{N}_{j}^{\infty}\right\} \quad \forall j \in \mathbb{N}_{0}^{p-1} .
\end{aligned}
$$

This states that the sets $\mathbb{C}_{j}^{*}$ constituting the maximum robust periodic controlled invariant set are the sets of all initial states $x_{j}$ such that there exists a control law $\kappa_{j}(\cdot)$, for each inter-period step index $j \in \mathbb{N}_{0}^{p-1}$, such that applying these control laws achieves constraint satisfaction indefinitely. To facilitate the use of the maximum robust periodic controlled invariant set in MPC problem design make Assumption 11.

Assumption 11: $\mathbb{C}_{j}^{*} \neq \emptyset \forall j \in \mathbb{N}_{0}^{p-1}$, i.e. the maximum robust periodic controlled invariant set exists.

Methods for the determination of maximum robust periodic controlled invariant sets are not provided in this paper. The determination of maximum robust periodic controlled invariant sets for a very similar problem setting was considered in [5]. The determination of maximum periodic controlled invariant sets for general linear periodic systems was considered in $[14,15]$. The maximum robust periodic controlled invariant sets of this paper can be computed with straightforward modifications of the methods presented in $[5,14,15]$. The interested reader is referred to $[4,8]$ for literature on the underlying set invariance tools for timeinvariant systems. For the remainder of this paper it is assumed that a method to determine the maximum robust periodic controlled invariant sets of Definition 10 is available.

\section{Least-Restrictive Robustly Strongly Feasible Periodic MPC Problems}

In this section the maximum robust periodic controlled invariant set $\left\{\mathbb{C}_{0}^{*}, \ldots, \mathbb{C}_{p-1}^{*}\right\}$ is employed to least-restrictively enforce robust strong feasibility of MPC Problem 3.

Let $\overline{\mathbb{C}}_{\bmod (j+1, p)}^{*}:=\mathbb{C}_{\bmod (j+1, p)}^{*} \ominus\left\{C_{j} w \mid w \in \mathbb{W}_{j}\right\}$ $\forall j \in \mathbb{N}_{0}^{p-1}$. For all $j \in \mathbb{N}_{0}^{p-1}$ it holds that $\left[x+C_{j} w \in\right.$ $\left.\mathbb{C}_{\bmod (j+1, p)}^{*} \forall w \in \mathbb{W}_{j}\right] \Longleftrightarrow\left[x \in \overline{\mathbb{C}}_{\bmod (j+1, p)}^{*}\right]$. Note that $\overline{\mathbb{C}}_{j}^{*} \subset \mathbb{C}_{j}^{*} \forall j \in \mathbb{N}_{0}^{p-1}$ because $0 \in \mathbb{W}_{j} \forall j \in \mathbb{N}_{0}^{p-1}$ by Assumption 1. Further note that $\overline{\mathbb{C}}_{j}^{*} \neq \emptyset \forall j \in \mathbb{N}_{0}^{p-1}$ by Definition 10 of $\mathbb{C}_{j}^{*}$ and due to Assumption 11.

Theorem 12: If $\mathbb{X}_{j}=\overline{\mathbb{C}}_{j}^{*}$ and $\mathbb{Y}_{j} \supseteq \mathbb{C}_{j}^{*} \forall j \in \mathbb{N}_{0}^{p-1}$ in MPC Problem 3, then the following three statements hold:

[a] $\mathbb{F}_{j}=\mathbb{C}_{j}^{*} \forall j \in \mathbb{N}_{0}^{p-1}$ for any prediction horizon length $N$ and stage cost matrices $Q_{j}$ and $R_{j}$.

[b] MPC Problem 3 is robustly strongly feasible according to Definition 7 for any prediction horizon length $N$ and stage cost matrices $Q_{j}$ and $R_{j}$.

[c] MPC Problem 3 is a least-restrictive robustly strongly feasible MPC problem according to Definition 8.

Proof: [a] The following holds for any $N, Q_{j}$ and $R_{j}$. We first show that $\mathbb{F}_{j} \subseteq \mathbb{C}_{j}^{*} \forall j \in \mathbb{N}_{0}^{p-1}$. Consider any $x_{i} \in \mathbb{F}_{j}$, i.e. for $x_{i}$ MPC Problem 3 is feasible. By constraints (4) and (5), $\exists u_{(i, 0)} \in \mathbb{R}^{m}$ s.t. $E_{j} x_{i}+G_{j} u_{(i, 0)} \leq W_{j} \wedge$ $x_{(i, 1)} \in \overline{\mathbb{C}}_{\bmod (i+1, p)}^{*}$. This implies that $x_{i} \in \mathbb{C}_{j}^{*}$. We next show that $\mathbb{F}_{j} \supseteq \mathbb{C}_{j}^{*} \forall j \in \mathbb{N}_{0}^{p-1}$. Consider any $x_{i} \in \mathbb{C}_{j}^{*}$. By the definitions of $\mathbb{C}_{j}^{*}$ and $\overline{\mathbb{C}}_{j}^{*}$ and by constraints (4) and (5), $\exists u_{(i, 0)} \in \mathbb{R}^{m}$ s.t. $E_{j} x_{i}+G_{j} u_{(i, 0)} \leq W_{j} \wedge$ $x_{(i, 1)} \in \overline{\mathbb{C}}_{\bmod (i+1, p)}^{*}$. This implies that $x_{(i, 1)} \in \mathbb{C}_{\bmod (i+1, p)}^{*}$. Therefore $\exists\left\{u_{(i, k)} \in \mathbb{R}^{m}\right\}_{k=1}^{N-1}$ s.t. $\left[E_{\bmod (i+k, p)} x_{(i, k)}+\right.$ $\left.G_{\bmod (i+k, p)} u_{(i, k)} \leq W_{\bmod (i+k, p)} \forall k \in \mathbb{N}_{1}^{N-1}\right] \wedge\left[x_{(i, k)} \in\right.$ $\left.\mathbb{C}_{\bmod (i+k, p)}^{*} \forall k \in \mathbb{N}_{2}^{N}\right]$. Thus constraint (6) is satisfied.

[b] The following holds for any $N, Q_{j}$ and $R_{j}$. Consider any $x_{i} \in \mathbb{F}_{j}=\mathbb{C}_{j}^{*}$. By constraints (4) and (5), any solution $U_{i} \in \mathbb{U}\left(x_{i}\right)$ achieves $E_{j} x_{i}+G_{j} u_{(i, 0)} \leq W_{j} \wedge x_{(i, 1)} \in$ $\overline{\mathbb{C}}_{\bmod (i+1, p)}^{*}$. Consequently $x_{i+1}=A_{j} x_{i}+B_{j} F U_{i}+C_{j} w_{i}+$ $c_{j} \in \mathbb{C}_{\bmod (i+1, p)}^{*}=\mathbb{F}_{\bmod (i+1, p)} \forall w_{i} \in \mathbb{W}_{j}$. By induction this holds recursively.

[c] By Definition 10 of $\mathbb{C}_{j}^{*}$, there does not exist a collection of control laws $\kappa_{j}: \mathbb{R}^{n} \rightarrow \mathbb{R}^{m} \forall j \in \mathbb{N}_{0}^{p-1}$ such that the domain of $\kappa_{j}$ is larger then $\mathbb{C}_{j}^{*}$ for any $j \in \mathbb{N}_{0}^{p-1}$, and furthermore such that constraints can be satisfied indefinitely. Thus by Theorem 12[a,b] it holds that MPC Problem 3 is a leastrestrictive robustly strongly feasible MPC problem according to Definition 8.

In the remainder of this paper we employ MPC Problem 3 
with prediction state constraints $\mathbb{X}_{j}=\overline{\mathbb{C}}_{j}^{*}$ and $\mathbb{Y}_{j}=\mathbb{C}_{j}^{*} \forall j \in$ $\mathbb{N}_{0}^{p-1}$. The choice of $\mathbb{X}_{j}$ in constraint (5) enforces robust strong feasibility. According to Theorem 12 any choice of $\mathbb{Y}_{j} \supseteq \mathbb{C}_{j}^{*} \forall j \in \mathbb{N}_{0}^{p-1}$, for example $\mathbb{Y}_{j}=\mathbb{R}^{n} \forall j \in \mathbb{N}_{0}^{p-1}$, results in MPC Problem 3 being a least-restrictive robustly strongly feasible MPC problem. The choice of $\mathbb{Y}_{j}$ above constrains the predicted nominal state trajectory to follow a path that is admissible in closed-loop. By constraining the predicted state trajectory to lie within the sets comprising the maximum robust periodic controlled invariant set, at each prediction step the predicted state satisfies a necessary and sufficient condition for the existence of an infinite control input trajectory such that applying this control input trajectory satisfies the constraints indefinitely. The finite-horizon MPC problem is thus expected to better approximate the infinitehorizon MPC problem than if $\mathbb{Y}_{j} \supset \mathbb{C}_{j}^{*} \forall j \in \mathbb{N}_{0}^{p-1}$.

Computing maximum controlled invariant sets is generally challenging, and the ability to determine the maximum robust periodic controlled invariant set limits the applicability of the proposed approach. Any non-maximum robust periodic controlled invariant set may be employed to enforce robust strong feasibility of MPC Problem 3, but may result in an MPC problem that is not least-restrictive.

\section{Building Climate Control}

A simplified building climate control problem plant is depicted in Fig. 1 [17].

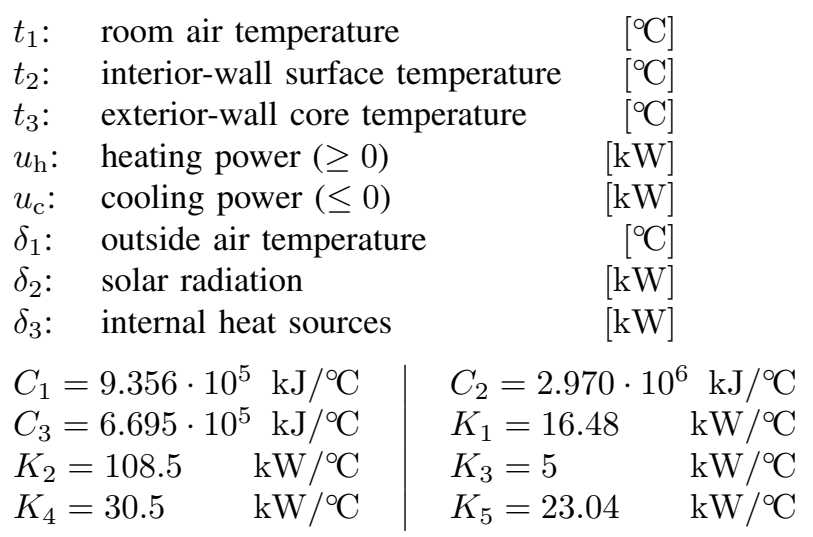

The system states are temperatures $t_{1}, t_{2}$ and $t_{3}$. The control inputs are $u_{\mathrm{h}}$ and $u_{\mathrm{c}}$. Environmental inputs $\delta_{1}, \delta_{2}$ and $\delta_{3}$ are assumed unaffected by the system. The two instances of $\delta_{2}$ in Fig. 1 signify that half the incident solar radiation heats the room directly via the windows, whereas the other half is absorbed by the exterior walls. The heat capacities of nodes with temperature $t_{1}, t_{2}$ and $t_{3}$ are $C_{1}, C_{2}$ and $C_{3}$, respectively. Heat capacities $C_{k}$ and gains $K_{k}$ for a specific

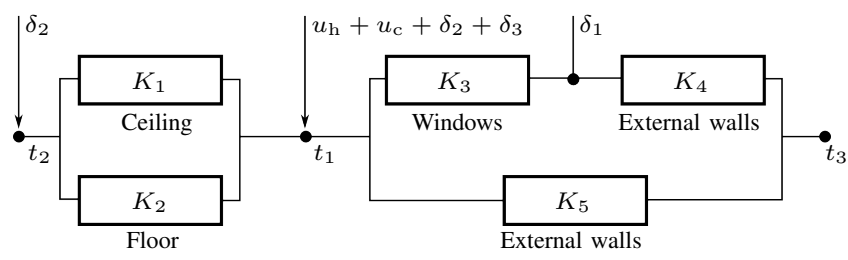

Fig. 1. Building climate control problem plant schematic. building and a room with $3600 \mathrm{~m}^{2}$ floor area were employed [17]. The continuous-time dynamics are as follows:

$$
\begin{aligned}
\dot{t}_{1}= & \frac{1}{C_{1}}\left[\left(K_{1}+K_{2}\right)\left(t_{2}-t_{1}\right)+K_{5}\left(t_{3}-t_{1}\right)\right. \\
& \left.\quad+K_{3}\left(\delta_{1}-t_{1}\right)+u_{\mathrm{h}}+u_{\mathrm{c}}+\delta_{2}+\delta_{3}\right] \\
\dot{t}_{2}= & \frac{1}{C_{2}}\left[\left(K_{1}+K_{2}\right)\left(t_{1}-t_{2}\right)+\delta_{2}\right] \\
\dot{t}_{3}= & \frac{1}{C_{3}}\left[K_{5}\left(t_{1}-t_{3}\right)+K_{4}\left(\delta_{1}-t_{3}\right)\right] .
\end{aligned}
$$

This is expressed as continuous-time LTI system

$$
\dot{x}=A_{\mathrm{c}} x+B_{\mathrm{c}} u+C_{\mathrm{c}} \omega
$$

with state $x=\left[t_{1}, t_{2}, t_{3}\right]^{\top} \in \mathbb{R}^{3}$, control input $u=\left[u_{\mathrm{h}}, u_{\mathrm{c}}\right]^{\top}$ $\in \mathbb{R}^{2}$, and environmental inputs $\omega=\left[\delta_{1}, \delta_{2}, \delta_{3}\right]^{\top} \in \mathbb{R}^{3}$.

Input constraints (9) are imposed at all times. Timedependent state constraints (10) and (11) are imposed so that during business hours the controller provides a comfortable work environment. Outside of business hours rooms are allowed to cool or warm to reduce energy consumption.

$$
\begin{aligned}
& 0 \leq u_{\mathrm{h}} \leq 200 \quad, \quad-50 \leq u_{\mathrm{c}} \leq 0 \\
& t_{1} \geq \begin{cases}21 & \text { from 8am to } 6 \mathrm{pm} \\
19 & \text { otherwise }\end{cases} \\
& t_{1} \leq \begin{cases}26 & \text { from } 8 \text { am to } 6 \mathrm{pm} \\
30 & \text { otherwise }\end{cases}
\end{aligned}
$$

Environmental input $\omega$ consists of two parts such that $\omega=d+w$, where $d$ is deterministic and periodically time-dependent with period length $24 \mathrm{~h}$, and $w$ is stochastic, bounded and subject to periodically time-dependent bounds with period length $24 \mathrm{~h}$. The deterministic input $d$ is employed to model known fluctuations in environmental conditions, for example that the sun rises in the morning and sets in the evening, and that most internal heat sources (office workers, equipment, etc.) $\delta_{3}$ are active from $8 \mathrm{am}$ to $6 \mathrm{pm}$. The stochastic input $w$ is employed to yield robustness against unknown environmental conditions, for example because
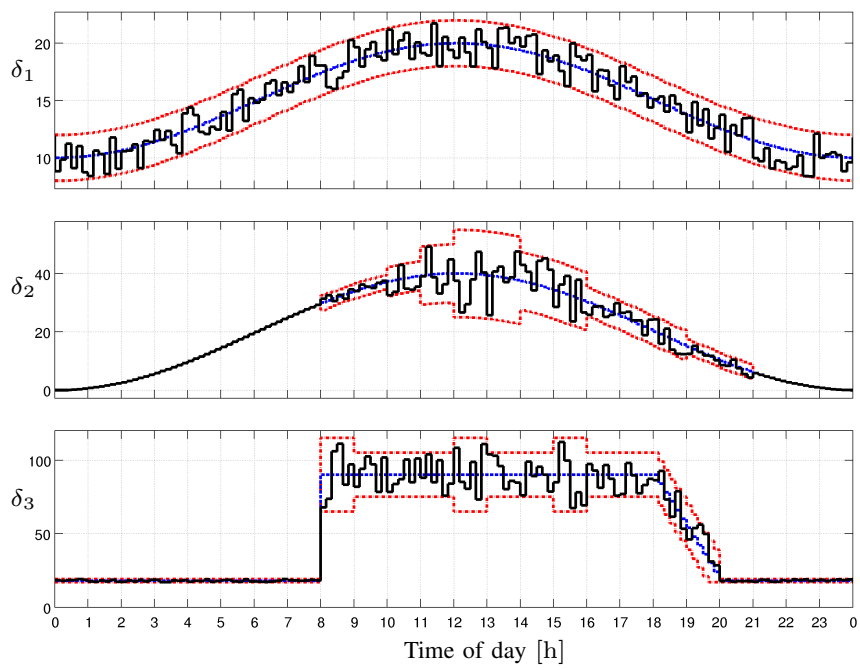

Fig. 2. Deterministic environmental inputs $d$ (dashed, blue), bounds on stochastic environmental inputs $w$ (dash-dotted, red), and realization of environmental inputs $\delta$ (solid, black) over one day. 

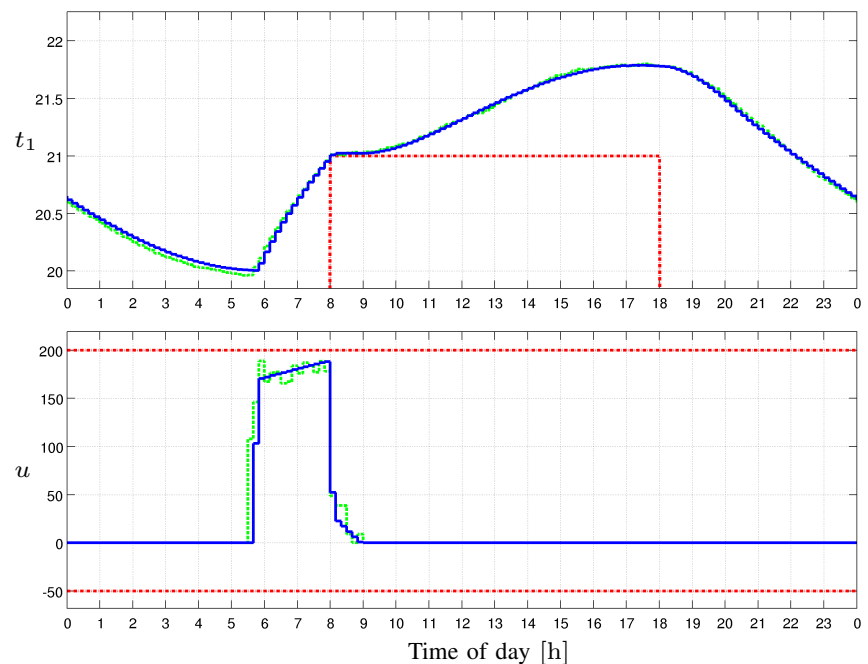

Fig. 3. Room temperature $t_{1}$ and heating/cooling input $u=u_{\mathrm{h}}+u_{\mathrm{c}}$ trajectories over one day; nominal (blue, solid), with stochastic disturbances (green, dashed), constraints (red, dash-dotted): $\kappa_{1}$.

cloud cover affects the amount of solar radiation $\delta_{2}$ reaching the building, and because an office worker may either heat her lunch in the office microwave and continue working (increasing $\delta_{3}$ ) or switch off her PC and go to a restaurant (reducing $\delta_{3}$ ). The bounds on stochastic input $w$ are assumed known, but the stochastic properties of $w$ are unknown.

The LTI dynamics of Eq. (8), constraints (9), (10) and (11), deterministic environmental inputs $d$ and the bounds on stochastic environmental inputs $w$ are sampled with a zero-order hold and a sample-period of 10min, resulting in periodic affine system (1) with period length $p=144$.

The time-discretized deterministic environmental inputs $d$, bounds on stochastic environmental inputs $w$ and a realization of environmental inputs $\omega$ are plotted in Fig. 2. Values for $d$ and bounds on $w$ are based on real data [17], but simplified. Stochastic inputs $w$ were randomly generated using a probability density function that is constant between the bounds, and zero otherwise. This stochastic input model does not reflect reality and is employed for simplicity.

The maximum robust periodic controlled invariant set was determined using the iterative algorithm of [5]. Computations were completed in 228 iterations and 79 seconds (average of 10 runs), and were performed on a $3.33 \mathrm{Ghz} \times 86-64$ processor running Matlab and the Multi-Parametric Toolbox [22]. Polytope projection was performed using Fourier elimination.

The state reference trajectory is $r_{j}=[22,0,0]^{\top} \forall j \in$ $\mathbb{N}_{0}^{p-1}$. A room temperature of $t_{1}=22^{\circ} \mathrm{C}$ is considered optimal (in this paper), whereas any value $21^{\circ} \mathrm{C} \leq t_{1} \leq 26^{\circ} \mathrm{C}$ is considered acceptable, for an office work environment.

Cost matrices $Q_{j}$ and $R_{j}$ are chosen as follows, where $q \geq 0$ is a scalar variable chosen later:

$$
\begin{aligned}
Q_{j} & = \begin{cases}\operatorname{diag}(q, 0,0) & \text { from } 8 \mathrm{am} \text { to } 6 \mathrm{pm} \\
0 & \text { otherwise }\end{cases} \\
R_{j} & = \begin{cases}{[2,-8]} & \text { from } 6 \mathrm{am} \text { to } 10 \mathrm{pm} \\
{[1,-4]} & \text { otherwise. }\end{cases}
\end{aligned}
$$
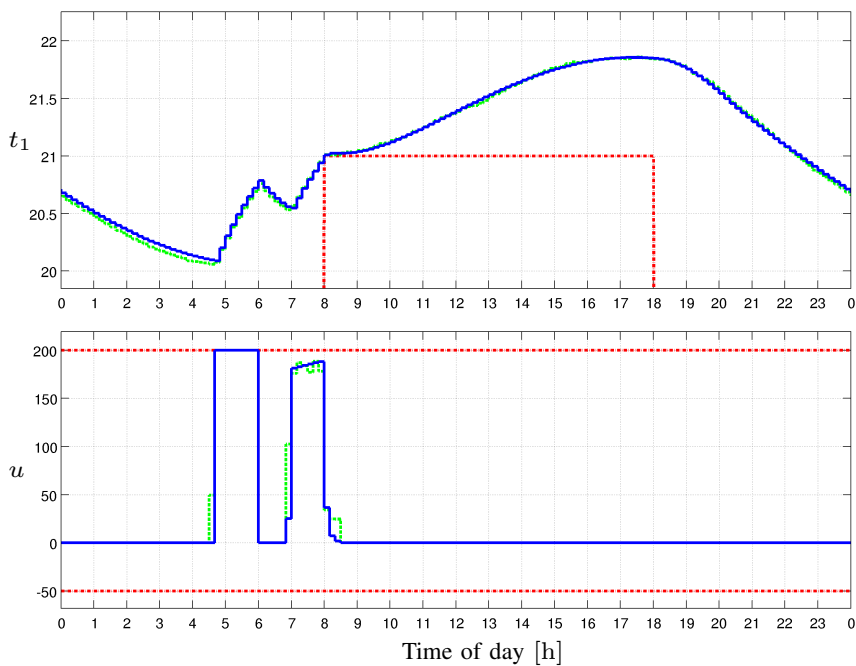

Fig. 4. Room temperature $t_{1}$ and heating/cooling input $u=u_{\mathrm{h}}+u_{\mathrm{c}}$ trajectories over one day; nominal (blue, solid), with stochastic disturbances (green, dashed), constraints (red, dash-dotted): $\kappa_{2}$.

The choice of $Q_{j}$ signifies that depending on the value of $q$ the deviation from the state-reference $r$ of $t_{1}$ is penalized during business hours, whereas deviations from the statereference $r$ of $t_{2}$ and $t_{3}$ are never penalized. The choice of $R_{j}$ models that energy prices during the day are twice those during the night. Simple high/low tariffs, also termed peak/off-peak and day/night tariffs, have existed for years in many European countries, e.g. France, Germany and Switzerland $[9,10,30]$. Furthermore the choice of $R_{j}$ reflects the fact that cooling is significantly (assumed four times) more expensive than heating, for the same power into/out of the room [17]. This is also reflected in constraints (9). Recall that $u_{\mathrm{c}}$ is negative, hence the minus sign in $R_{j}$.

The four control laws with details tabulated in Table I are considered. The trajectories of room temperature $t_{1}$ and heating/cooling input $u_{\mathrm{h}}+u_{\mathrm{c}}$ due to each control law for one day are plotted in the listed figures. The one-day disturbance pattern of Fig. 2 was applied over the one-day trajectories plotted in Figs. 3-6. Denote by

$$
\begin{aligned}
\bar{t}_{1} & :=\frac{1}{p} \sum_{i=0}^{p-1}[1,0,0] x_{i} \\
\bar{u} & :=\frac{1}{p} \sum_{i=0}^{p-1}[1,-4] \kappa\left(x_{i}\right) \\
\bar{V} & :=\frac{1}{p} \sum_{i=0}^{p-1}\left[\mathcal{L}_{\mathrm{x}}\left(x_{i}, i\right)+\mathcal{L}_{\mathrm{u}}\left(\kappa\left(x_{i}\right), i\right)\right]
\end{aligned}
$$

the average room temperature $t_{1}$, power consumption (in

TABLE I. Controller settings and results: Prediction horizon length $N$, room temperature error cost $q$, Figure number, and average room temperature $\bar{t}_{1}\left[{ }^{\circ} \mathrm{C}\right]$, average heating/cooling power consumption $\bar{u}[\mathrm{~kW}]$ and average objective cost $\bar{V}$, over one day for the nominal case.

\begin{tabular}{lcccccr} 
Controller & $N$ & $q$ & Fig. & $\bar{t}_{1}$ & $\bar{u}$ & \multicolumn{1}{c}{$\bar{V}$} \\
\hline$\kappa_{1}$ & 1 & 0 & 3 & 21.0 & 17.7 & 33.5 \\
$\kappa_{2}$ & 72 & 0 & 4 & 21.1 & 19.3 & 27.5 \\
$\kappa_{3}$ & 1 & $10^{6}$ & 5 & 21.3 & 51.0 & 24241 \\
$\kappa_{4}$ & 72 & $10^{6}$ & 6 & 21.6 & 96.9 & 572
\end{tabular}



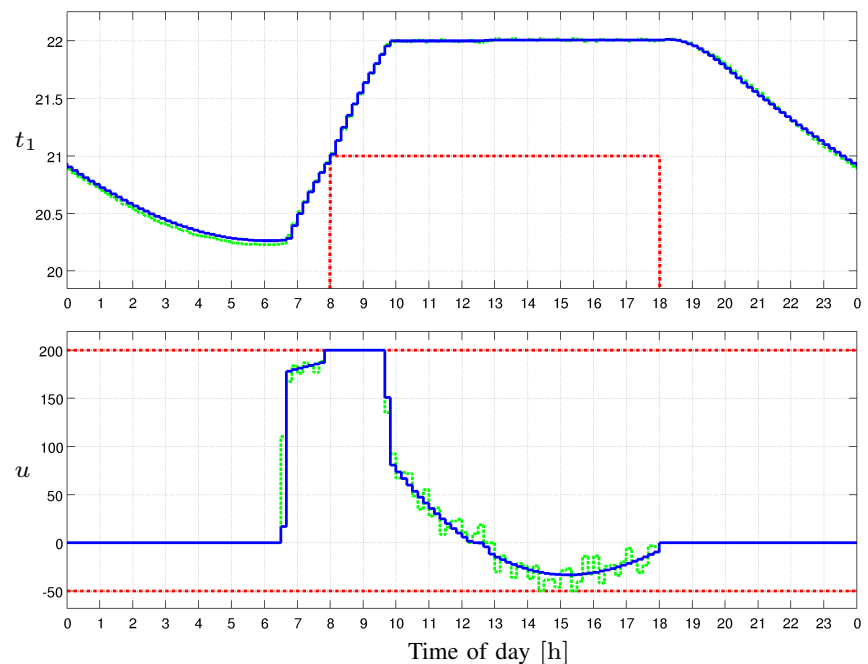

Fig. 5. Room temperature $t_{1}$ and heating/cooling input $u=u_{\mathrm{h}}+u_{\mathrm{c}}$ trajectories over one day; nominal (blue, solid), with stochastic disturbances (green, dashed), constraints (red, dash-dotted): $\kappa_{3}$.

$\mathrm{kW})$ and objective cost over the one-day period plotted in Figs. 3-6, respectively, for the nominal case.

Control laws $\kappa_{1}$ and $\kappa_{2}$ have a state-error cost of zero. Thus these control laws aim to minimize the energy cost. Room temperature has no effect on the control objective, except that all constraints must be satisfied. Control laws $\kappa_{1}$ and $\kappa_{2}$ differ only in the prediction horizon length, $10 \mathrm{~min}$ vs. $12 \mathrm{~h}$. The explicit use of the maximum robust periodic controlled invariant set of Definition 10 in MPC Problem 3 to least-restrictively enforce robust strong feasibility allows the use of any prediction horizon length, while guaranteeing constraint satisfaction. Thus despite the short prediction horizon, prediction constraint (5) in MPC Problem 3 provides control law $\kappa_{1}$ with all the required information to be able to impose the minimum admissible room temperature at all times. Thus, effectively, $\kappa_{1}$ is the minimum energy control law, where energy refers to heating/cooling energy in kJ, not the signal energy of control input signal $u$. Due to the long prediction horizon control law $\kappa_{2}$ can exploit cheaper energy prices before $6 \mathrm{am}$. Thus control law $\kappa_{2}$ achieves a lower objective cost $\bar{V}$ than control law $\kappa_{1}$, but at a higher average power consumption.

Control laws $\kappa_{3}$ and $\kappa_{4}$ attempt to regulate the room temperature $t_{1}$ to $22^{\circ} \mathrm{C}$ from $8 \mathrm{am}$ to $6 \mathrm{pm}$, with little regard for power consumption. They differ from each other in the prediction horizon length, $10 \mathrm{~min}$ vs. $12 \mathrm{~h}$. Similarly to control law $\kappa_{1}$, control law $\kappa_{3}$ begins heating the room as late as possible, due only to constraint (5) and the use of the maximum robust periodic controlled invariant set in MPC Problem 3, for the purpose of achieving $t_{1} \geq 21$ at $8 \mathrm{am}$. At 8 am control law $\kappa_{3}$ continues heating to achieve $t_{1}=22^{\circ} \mathrm{C}$. In contrast, the long prediction horizon of control law $\kappa_{4}$ allows heating to come on much earlier than with control law $\kappa_{3}$, and the desired room temperature of $22^{\circ} \mathrm{C}$ is achieved successfully during business hours.
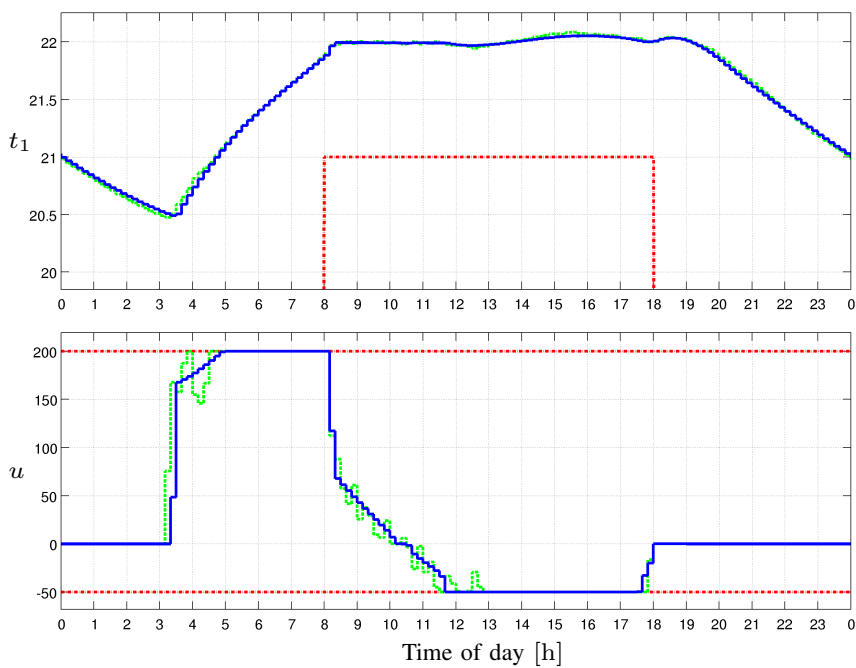

Fig. 6. Room temperature $t_{1}$ and heating/cooling input $u=u_{\mathrm{h}}+u_{\mathrm{c}}$ trajectories over one day; nominal (blue, solid), with stochastic disturbances (green, dashed), constraints (red, dash-dotted): $\kappa_{4}$.

\section{CONCLUSION}

Maximum robust periodic controlled invariant sets for periodic affine systems with bounded, additive disturbances were characterized and employed in the design of leastrestrictive robustly strongly feasible periodic MPC problems. In many applications, for example the problem of room temperature control using a minimum amount of energy considered in this paper, the control performance is directly related to the ability to operate close to the constraints, while rigorously enforcing constraint satisfaction indefinitely. The use of maximum controlled invariant sets is indispensable in these situations, as they yield necessary and sufficient conditions for the existence of a control input such that all constraints can be satisfied in the future. The use of maximum periodic controlled invariant sets is indispensable in the design of least-restrictive periodic MPC controllers.

The MPC problem formulation follows the common nominal open-loop prediction paradigm. By suitably tailoring the prediction state constraints for each prediction step, instead of usual terminal constraints, both the computational complexity of closed-loop prediction MPC formulations, as well as the conservativeness of min-max open-loop prediction MPC formulations, were straightforwardly circumnavigated.

Periodic affine systems provide a powerful modeling framework for a large variety of control problems, yet can be dealt with using nearly standard methods. The ability to model not only periodic dynamics, but also periodic constraints, disturbances, disturbance bounds, external input variations, etc., is extremely powerful. The example of building climate control was used to convey the flexibility and convenience of the proposed approach. Although the dynamics of the plant are time-invariant, incorporating the known, periodically time-dependent constraints and disturbance bounds into a time-invariant MPC framework would be at best conservative. 


\section{REFERENCES}

[1] Bemporad, A., Reducing Conservativeness in Predictive Control of Constrained Systems with Disturbances, Proc. ACC, USA, 1998.

[2] Bemporad, A., \& Morari, M, Robust Model Predictive Control: A Survey, Robustness in Identification and Control, Vol. 245, pp. 207226, 1999.

[3] Bittanti, S., Colaneri, P., Periodic Systems Filtering and Control, Springer, 2009.

[4] Blanchini, F., Set Invariance in Control, Automatica, Vol. 35, pp. 17471767, 1999.

[5] Blanchini, F., \& Ukovich, W., Linear Programming Approach to the Control of Discrete-Time Periodic Systems with Uncertain Inputs J. Optim. Theory and Applications, Vol. 78, No. 3, pp. 523-539, 1993.

[6] Böhm, C., Raff, T., Reble, M., Allgöwer, F., LMI-Based Model Predictive Control for Linear Discrete-Time Periodic Systems, Lecture Notes in Control and Information Sciences, Vol. 384, Springer, 2009.

[7] Böhm, C., Yu, S., Allgöwer, F., Predictive control for constrained discrete-time periodic systems using a time-varying terminal region, Proc. Conf. Methods and Models in Automation and Robotics, Poland, 2009.

[8] Dória, C. E. T., \& Hennet, J. C., (A, B)-Invariant Polyhedral Sets of Linear Discrete-Time Systems, J. Optimization Theory and Applications, Vol. 103, No. 3, pp. 521-542, 1999.

[9] EDF Tariffs. www. edf-bleuciel. fr, accessed March 10, 2010.

[10] EWZ Details of end-consumer electricity tariffs. Www. stadt-zuer ich.ch/content/ewz/de/index.html/energie/stromp roduktezuerich/privatkunden/ewz $\% 20$ naturpower $\% 20$ privatkunden/preiskomponenten.html, accessed March 10, 2010.

[11] Fukushima, H., \& Bitmead, R. R., Robust Constrained Model Predictive Control using Closed-loop Prediction, Proc. ACC, USA, 2003.

[12] Gondhalekar, R., Imura, J., \& Kashima, K., Controlled invariant feasibility - A general approach to enforcing strong feasibility in MPC applied to move-blocking, Automatica, Vol. 45, No. 12, pp. 2869-2875, 2009.

[13] Gondhalekar, R., \& Imura, J. Least-Restrictive Move-Blocking Model Predictive Control, Automatica, Vol. 46, No. 7, pp. 1234-1240, 2010.

[14] Gondhalekar, R., \& Jones, C. N., Model Predictive Control of Linear Periodic Systems - A Unified Framework Including Control of Multirate and Multiplexed Systems, Proc. 48th CDC, China, 2009.

[15] Gondhalekar, R., \& Jones, C. N., MPC of Constrained Discrete-Time Linear Periodic Systems - A Framework for Asynchronous Control: Strong Feasibility, Stability and Optimality via Periodic Invariance, Automatica, provisionally accepted, based on [14].
[16] Goulart, P. J., Kerrigan, E. C., \& Maciejowski, J. M, Optimization over state feedback policies for robust control with constraints, Automatica, Vol. 42, No. 4, pp. 523-533, 2006.

[17] Gwerder, M., \& Tödtli, J., Predictive Control for Integrated Room Automation, 8th REHVA World Congress for Building Technologies - CLIMA 2005, Switzerland, 2005.

[18] Henze, G. P., Kalz, D., Liu, S. \& Felsmann, C., Experimental analysis of model-based predictive optimal control for active and passive building thermal storage inventory, Int. J. of HVAC\&R Research, Vol. 11, No. 2, pp. 189-214, 2005.

[19] International Energy Agency, Energy efficiency requirements in building codes, energy efficiency policies for new buildings, www. iea. org/publications/free_new_Desc. asp?PUBS_ID =2042.

[20] Kern, B., Böhm, C., Findeisen, R., Allgöwer, F., Receding Horizon Control for Linear Periodic Time-Varying Systems Subject to Input Constraints, Lecture Notes in Control and Information Sciences, Vol. 384, Springer, 2009.

[21] Kim, K. B., Lee, J-W., Kwon, W. H., Intervalwise Receding Horizon $H_{\infty}$ Tracking Control for Discrete Linear Periodic Systems, IEEE Trans. Aut. Control, Vol. 45, No. 4, pp. 747-752, 2000.

[22] Kvasnica, M., Grieder, P., Baotić, M., Multi-Parametric Toolbox (MPT), http://control.ee.ethz.ch/ mpt/, 2004

[23] Lee, J. H., Natarajan, S., Lee, K. S., A model-based predictive control approach to repetitive continuous processes with periodic operations, J. Process Control, Vol. 11, pp. 195-207, 2001.

[24] Löfberg, J., Approximations of closed-loop minimax MPC, Proc. 42nd CDC, USA, 2003.

[25] Lovera, M., Varga, A., Optimal discrete-time magnetic attitude control of satellites, Proc. 16th IFAC World Congress, Czech Republic, 2005.

[26] Meyer, R. A., Burrus, C. S., A Unified Analysis of Multirate and Periodically Time-Varying Digital Filters, IEEE Trans. Circuits and Systems, Vol. CAS-22, No. 3, pp. 162-168, 1975.

[27] Oldewurtel, F., Parisio, A., Jones, C. N., Morari, M., Gyalistras, D., Gwerder, M., Stauch, V., Lehmann, B., \& Wirth, K. Energy Efficient Building Climate Control using Stochastic Model Predictive Control and Weather Predictions, Proc. ACC, USA, 2010.

[28] Reble, M., Böhm, C., Allgöwer, F., Nonlinear Model Predictive Control for Periodic Systems Using LMIs, Proc. 10th ECC, Hungary, 2009.

[29] Varga, A., An overview of recent developments in computational methods for periodic systems, Proc. 3rd IFAC Workshop on Periodic Control Systems, Russia, 2007.

[30] Vattenfall Europe AG End consumer Tariffs. Berlin Basis Privat-strom, www. vattenfall. de/www/vf/vf_de/202436priva/2024 96produ/704818berli/index.jsp, accessed March 10, 2010. 